\title{
High Dimension Model Representation-Based Response Surface for Reliability Analysis of Tunnel
}

\author{
Hongbo Zhao \\ School of Civil Engineering, Henan Polytechnic University, Jiaozuo 454003, China \\ Correspondence should be addressed to Hongbo Zhao; bxhbzhao@hotmail.com
}

Received 16 August 2018; Revised 9 October 2018; Accepted 16 October 2018; Published 7 November 2018

Academic Editor: Ramon Sancibrian

Copyright (c) 2018 Hongbo Zhao. This is an open access article distributed under the Creative Commons Attribution License, which permits unrestricted use, distribution, and reproduction in any medium, provided the original work is properly cited.

\begin{abstract}
Uncertainty is an important prosperity to rock tunnel. Reliability analysis is widely used to deal with the uncertainty. But it is difficult to be adopted in rock tunnel using the traditional reliability method because the limit state function is an implicit function. High dimension model representation (HDMR) can approximate the high dimensional, nonlinear, and implicit function using the low dimensional function. In this study, the HDMR method was adapted to approximate the limit state function through combining with response surface method (RSM). A new reliability analysis approach of HDMR-based response surface method, combined with the first-order reliability method (FORM), is developed to calculate the reliability index of tunnel, and implementation of the method is explained briefly. A circular tunnel with analytical solution and horseshoe tunnel with numerical solution are used to demonstrate the proposed method. The obtained reliability index is in excellent agreement with Low and Tang's (2007) method and traditional RSM. It shows that HDMR-based response surface can approximate well the limit state function, and the proposed method is an efficient and effective approach for reliability analysis in tunnel engineering. It is very useful for reliability analysis of practical large-scale rock engineering.
\end{abstract}

\section{Introduction}

Stability analysis of tunnel is very important to optimization design and safety construction. Various methods such as analytical method and numerical method are widely used to estimate the stability of rock tunnel in the past decades. But there exists a lot of uncertainty in tunnel engineering which affects the design and construction. Traditional numerical method cannot consider the uncertainty in design and construction of tunnel. It restricts the application of numerical method, and that brings the risk for safety construction. Reliability analysis, which is commonly applied in engineering systems, provides a good way to deal with the uncertainty.

In recent years, reliability analysis of tunnels was paid more attention by many researchers [1-8]. Based on analytical solutions, Hoek (1998) estimated the reliability of circular tunnels by Monte Carlo simulation using @RISK commercial software [1]. Oreste (2005) applied a probabilistic numerical approach to the design of primary tunnel supports [3]. Li and Low (2010) estimated the reliability of circular tunnels by combining an analytical solution and first-order reliability method (FORM) [6]. Mollon et al. $(2009,2011)$ studied the reliability of rock tunnel stability using a numerical model $[4,5]$. Lü and Low $(2011)$ and Lü et al. $(2011,2017)$ performed a probabilistic analysis of underground rock excavation and of ground-support interaction of tunnel using the response surface method [7-9]. Su et al. (2011) presented a practical algorithm for FORM to deal with the implicit nature of a limit state function. But there exists lots of difficulty in applying reliability analysis to practical rock tunnel [10].

With the development of computational intelligence, artificial neural network (ANN) and support vector machine (SVM) have been used in the reliability analysis of engineering system. Deng et al. (2005) proposed an ANNbased second-order reliability method and an ANN-based Monte Carlo simulation method [2]. Hosnielhewy et al. (2006) proposed an ANN-based response surface method to analyze the reliability of structures [11]. Other researchers have applied ANN to reliability analysis by combining it with Monte Carlo simulation, FORM, response surface method, etc. [12-15]. SVM was adopted to approximate the limit state function and calculate the reliability index of tunnel through 
combining with first-order reliability method (FORM) and Monte Carlo simulation (MCS) $[16,17]$. But ANN and SVM need more training samples to construct a model. It is difficult to obtain sufficient training samples for practical engineering because of the complexity, cost, and efficiency of problem. And the performance of generalization of ANN and SVM also restricted the application in practical engineering.

High dimensional model representation (HDMR) is a general set of quantitative model assessment and analysis tools for capturing the nonlinear, implicit, and high dimensional relationships between sets of input and output variables [18]. HDMR can approximate the implicit and high dimensional limit state function using lower dimensional function. In this study, the HDMR was adopted to approximate the limit state function, and then FORM was used to compute the reliability index of tunnels. The coefficient of HDMR model was determined using moving least square method. The remainder of this study is structured as follows. In Section 2, the FORM algorithm is reviewed in briefly. Section 3 introduces the basic idea of RSM and HDMR, the HDMR-based response surface is proposed, and the procedure of the proposed reliability analysis was explained in detail. In Section 4, the proposed method was applied to a circular tunnel with analytical solution and a horseshoe tunnel using numerical solution. The results were compared with FORM and traditional RSM. Some conclusions are given in Section 5.

\section{First-Order Reliability Method (FORM)}

FORM is widely used in reliability analysis. In this study, we use a practical FORM method, which was presented by Low (2004) based on the Hasofer-Lind index [19, 20]. To obviate the computations of equivalent normal means and equivalent normal standard deviations, Low and Tang (2007) proposed a new efficient algorithm for the FORM via varying dimensionless number $n_{i}$ to estimate the reliability index as follow [21]:

$$
\beta=\min _{X \in F} \sqrt{[n]^{T}[R]^{-1}[n]}
$$

where $\mathbf{n}$ is a column vector of $n_{\mathrm{i}}$. $\mathrm{R}$ is the correlation matrix. Once obtaining the reliability index, the probability of failure can be evaluated from

$$
p_{f} \approx 1-\Phi(\beta)
$$

In this study, (1) was adopted to compute the reliability analysis based on FORM proposed by Low and Tang.

\section{HDMR-Based Response Surface Method}

To the reliability analysis of practical tunnel engineering, the limit state function is implicit, high dimensional, nonlinear function. An important part of reliability analysis is to develop an efficient method to approximate the above limit state function. Response surface method is a commonly used method which adopts a polynomial to represent the limit state function based on numerical simulation. Practically for the most engineering systems, only relatively low order correlations of the input variables are expected to have a significant effort on the response. HDMR utilizes the above property to represent the high dimensional relationships between sets of input and output model variables using the low dimensional hierarchical function.

3.1. High Dimension Model Representation (HDMR). Let the $\mathrm{N}$-dimensional vector $x=\left\{x_{1}, x_{2}, \ldots x_{\mathrm{N}}\right\}$ represent the input variables of the model under consideration and the response variables as $g(x)$. According to HDRM model [22], the response $g(x)$ can be expressed as a hierarchical correlated function in terms of the input variables as

$$
\begin{aligned}
\mathrm{g}(\mathrm{x})= & \mathrm{g}_{0}+\sum_{i=1}^{N} g_{i}\left(x_{i}\right)+\sum_{1 \ll i_{1} \ll i_{2} \ll N} g_{i_{1} i_{2}}\left(x_{i_{1}}, x_{i_{2}}\right) \\
& +\sum_{1 \ll i_{1} \cdots \ll i_{l} \ll N} g_{i_{1} i_{2} \ldots i_{l}}\left(x_{i_{1}}, x_{i_{2}}, \ldots, x_{i_{l}}\right) \\
& +g_{12 \ldots N}\left(x_{1}, x_{2}, \ldots, x_{N}\right)
\end{aligned}
$$

where $g_{0}$ is a constant term representing the 0th order component function of $g(x), g_{i}\left(x_{i}\right)$ is a 1st order term of $g(x)$ which represents the effect of only variables $x_{i} \cdot g_{\mathrm{i} 1 \mathrm{i} 2}\left(x_{i 1}\right.$, $x_{i 2}$ ) is the 2 nd term that represents the cooperative effects of the variables $x_{i 1}, x_{i 2} \cdot g_{i 2, \ldots, \mathrm{N}}\left(x_{1}, x_{2}, \ldots, x_{\mathrm{N}}\right)$ contains any residual dependence of all the relevant component functions in (3). Once all component functions are determined in (3), the component function constitutes HDMR. Generally, the high order component is negligible, so HDMR can be used to approximate the $g(x)$ with high efficiency.

There are two methods to determine the component functions. In this study, cut-HDMR is adopted to determine the component functions in (3) which approximate the implicit limit state function in reliability analysis. Based on cut-HDMR procedure [23], the first- and second-order approximations of $\mathrm{g}(\mathrm{x})$ are denoted, respectively, by

$$
\begin{aligned}
\tilde{g}(x)= & \sum_{i=1}^{N} g\left(c_{1}, \ldots, c_{i-1}, x_{i}, c_{i+1}, \ldots, c_{N}\right) \\
& -(N-1) g(c)
\end{aligned}
$$

and

$$
\begin{gathered}
\widetilde{g}(x)=\sum_{\substack{i_{1}=1, i_{2}=1 \\
i_{1}<i_{2}}}^{N} g\left(c_{1}, \ldots, c_{i_{1}-1}, x_{i_{1}}, c_{i_{1}+1}, \ldots, c_{i_{2}-1}, x_{i_{2}}, c_{i_{2}+1},\right. \\
\left.\ldots c_{N}\right)-(N-2) \sum_{i=1}^{N} g\left(c_{1}, \ldots, c_{i-1}, x_{i}, c_{i+1}, \ldots, c_{N}\right) \\
+\frac{(N-1)(N-2)}{2} g(c)
\end{gathered}
$$

where $c=\left(c_{1}, c_{2}, \ldots, c_{\mathrm{N}}\right)$ is a reference point which is a mean value of the input variables. $g\left(c_{1}, c_{2}, \ldots, c_{i-1}, x_{i}, c_{i+1}, \ldots, c_{\mathrm{N}}\right)$ denotes that all the input variables are at their reference point values except $x_{i} \cdot g(c)$ is the response of system evaluated 
at the reference point $c . g\left(c_{1}, c_{2}, c_{i 1-1}, x_{i 1}, c_{i 1+1}, \ldots c_{i 2-1}, x_{i 2}\right.$, $\left.c_{i 2+1}, \ldots, x_{\mathrm{N}}\right)$ is evaluated in a plane defined by the binary set of input variables $x_{i 1}, x_{i 2}$ through the reference point, etc.

The response of tunnel $g(x)$ at point $x$ can be obtained by the following two steps:

Step 1. Interpolate each of the low dimensional HDMR terms with respect to the input values of the point $x$. To the 1st and 2nd order component function in HDMR model, $n$ and $n^{2}$ function values were computed based on numerical simulation, i.e.,

$$
\begin{aligned}
& g\left(x_{i}^{j}\right)=g\left(c_{1}, \ldots, c_{i-1}, x_{i}^{j}, c_{i+1}, \ldots, c_{N}\right), \\
& j=1,2, \ldots, n \\
& g\left(x_{i 1}^{j 1}, x_{i 2}^{j 2}\right) \\
& =g\left(c_{1}, \ldots, c_{i_{1}-1}, x_{i 1}^{j 1}, c_{i_{1}+1}, \ldots, c_{i_{2}-1}, x_{i 2}^{j 2}, c_{i_{2}+1}, \ldots c_{N}\right), \\
& j_{1}=1,2, \ldots, n j_{2}=1,2, \ldots, n
\end{aligned}
$$

Central sample method was used to construct $x_{i}^{j}(j=1,2, \ldots, n)$ and $x_{i 1 j 1}, x_{i 2 j 2}$. Once obtaining $n$ and $n^{2}$ function values, the 1st and 2 nd component function can be obtained as

$$
\begin{aligned}
& g\left(x_{i}\right)=\sum_{i=1}^{n} \varphi_{j}\left(x_{i}\right) g\left(c_{1}, \ldots, c_{i-1}, x_{i}^{j}, c_{i+1}, \ldots, c_{N}\right) \\
& g\left(x_{i 1}, x_{i 2}\right)=\sum_{i_{1}=1 i_{2}=1}^{n} \sum_{j_{1 j_{2}}}^{n}\left(x_{i_{1}}, x_{i_{2}}\right) \\
& \quad \cdot g\left(c_{1}, \ldots, c_{i_{1}-1}, x_{i 1}^{j 1}, c_{i_{1}+1}, \ldots, c_{i_{2}-1}, x_{i 2}^{j 2}, c_{i_{2}+1}, \ldots c_{N}\right)
\end{aligned}
$$

where $\varphi_{j}\left(x_{i}\right)$ and $\varphi_{j 1 j 2}\left(x_{i 1}, x_{i 2}\right)$ can be obtained using moving least square method. The same procedure shall be repeated for all the 1st and 2 nd component functions, i.e., $g_{i}\left(x_{i}\right)$ and $g_{i 1 i 2}\left(x_{i 1}, x_{i 2}\right), i, i 1, i 2=1,2, \ldots, \mathrm{N}$.

Step 2. Sum the interpolated values of HDMR terms from zeroth order to highest order retained in keeping with the desired accuracy. The response surface of $g(x)$ based on HDMR can be obtained as

$$
\begin{aligned}
\tilde{g}(x)= & \sum_{i=1}^{N} \sum_{j=1}^{n} \varphi_{j}\left(x_{i}\right) g\left(c_{1}, \ldots, c_{i-1}, x_{i}^{j}, c_{i+1}, \ldots, c_{N}\right) \\
& -(N-1) g_{0}
\end{aligned}
$$

and

$$
\begin{aligned}
& \widetilde{g}(x)=\sum_{\substack{i_{1}=1, i_{2}=1 \\
i_{1}<i_{2}}}^{N} \sum_{i_{1}=1 i_{2}=1}^{n} \sum_{i_{1}}^{n} \varphi_{j_{1}}\left(x_{i_{1}}, x_{i_{2}}\right) \\
& \cdot g\left(c_{1}, \ldots, c_{i_{1}-1}, x_{i 1}^{j 1}, c_{i_{1}+1}, \ldots, c_{i_{2}-1}, x_{i 2}^{j 2}, c_{i_{2}+1}, \ldots c_{N}\right) \\
& -(N-2) \sum_{i=1}^{N} \sum_{j=1}^{n} \varphi_{j}\left(x_{i}\right) g\left(c_{1}, \ldots, c_{i-1}, x_{i}^{j}, c_{i+1}, \ldots, c_{N}\right) \\
& +\frac{(N-1)(N-2)}{1} g_{0}
\end{aligned}
$$

3.2. Moving Least Square Method. Consider that a function $u(x)$ can be approximated using the following form according to moving least square method [24]:

$$
u^{h}(x)=\sum_{i=1}^{m} p_{i}(x) a_{i}(x)=p^{T}(x) a(x)
$$

where $p^{T}(x)$ is a vector of complete basis function of order $\mathrm{m}$ and $a(x)$ is a vector or unknown parameter that depends on $x$.

The coefficient vector $a(x)$ can be determined by minimizing a weight function, defined as

$$
\begin{aligned}
J(\mathrm{x}) & =\sum_{I=1}^{n_{t}} w_{I}(x)\left[p^{T}\left(x_{I}\right) a(x)-d_{I}\right]^{2} \\
& =[P a(x)-d]^{T} W[P a(x)-d]
\end{aligned}
$$

where $x_{\mathrm{I}}$ denotes the coordinates of sample point I, $d \mathrm{~T}$ with $d$ I representing the nodal parameter for sample point $I$, $W$ denoting the weight function associated with sample point I. The stationarity of $J(x)$ with respect to $a(x)$ yields

$$
A(x) a(x)=C(x) d
$$

where

$$
\begin{aligned}
& \mathrm{A}(\mathrm{x})=\sum_{I=1}^{n_{t}} w_{I}(x) p\left(x_{I}\right) p^{T}\left(x_{I}\right)=P^{T} W P \\
& \mathrm{C}(\mathrm{x})=\left[w_{I}(x) p\left(x_{1}\right), \ldots, w_{n}(x) p\left(x_{n}\right)\right]=P^{T} W
\end{aligned}
$$

$a(x)$ can be obtained by solving the above equation and the approximated function $u^{h}(x)$ will be given

$$
u^{h}(x)=\sum_{I=1}^{n_{t}} \varphi_{I}(x) d_{I}=\varphi^{T}(x) d
$$

where

$$
\begin{aligned}
\varphi^{T}(x) & =\left\{\varphi_{1}(x), \varphi_{2}(x), \ldots, \varphi_{n_{t}}(x)\right\} \\
& =p^{T}(x) A^{-1}(x) C(x) \\
\varphi_{I}(x) & =\sum_{j=1}^{m} p_{j}(x)\left[A^{-1}(x) C(x)\right]_{j I}
\end{aligned}
$$


Weight function is important to the performance of HDMR. Lü et al. compared the performance of weight function in different parameters of Gaussian function [25]. To my knowledge, there is no rigorous theory to determine the weight function. In this study, Gaussian function was adopted in Eq. (17).

$$
W(r)= \begin{cases}\frac{e^{-\alpha^{2} r^{2}}-e^{-\alpha^{2}}}{1-e^{-\alpha^{2}}}, & r \leq 1 \\ 0, & r>1\end{cases}
$$

where $\alpha$ is the coefficient for controlling the shape of the weight function.

3.3. HDMR-Based Response Surface. In this study, HDMR is adopted to approximate the response of tunnel. Based on the HDMR, the response surface can be expressed as

$$
g(\boldsymbol{X})=\operatorname{HDMR}(\boldsymbol{X})
$$

where $g(\boldsymbol{X})$ is the limit state function and $\boldsymbol{X}$ represents the vectors of the random variables such as rock mechanical parameters and in situ stress. If the 1st and 2nd order component functions were adopted, the limit state function will be in the following form:

$$
\begin{aligned}
\operatorname{HDMR}(X) & \\
= & \sum_{i=1}^{N} \sum_{j=1}^{n} \varphi_{j}\left(x_{i}\right) g\left(c_{1}, \ldots, c_{i-1}, x_{i}^{j}, c_{i+1}, \ldots, c_{N}\right) \\
& \quad-(N-1) g_{0}
\end{aligned}
$$

And

$$
\begin{aligned}
& \operatorname{HDMR}(X)=\sum_{\substack{i_{1}=1, i_{2}=1 \\
i_{1}<i_{2}}}^{N} \sum_{i_{1}=1 i_{2}=1}^{n} \sum_{i_{1}}^{n} \varphi_{j_{1}}\left(x_{i_{1}}, x_{i_{2}}\right) \\
& \cdot g\left(c_{1}, \ldots, c_{i_{1}-1}, x_{i 1}^{j 1}, c_{i_{1}+1}, \ldots, c_{i_{2}-1}, x_{i 2}^{j 2}, c_{i_{2}+1}, \ldots c_{N}\right) \\
& -(N-2) \sum_{i=1}^{N} \sum_{j=1}^{n} \varphi_{j}\left(x_{i}\right) g\left(c_{1}, \ldots, c_{i-1}, x_{i}^{j}, c_{i+1}, \ldots, c_{N}\right) \\
& +\frac{(N-1)(N-2)}{1} g_{0}
\end{aligned}
$$

In this study, $\varphi_{j}\left(x_{i}\right)$ and $\varphi_{j 1 j 2}\left(x_{i 1}, x_{i 2}\right)$ can be obtained using moving least square method (see (14)-(19)). Central samples are used to determine $2 n+1$ samples, i.e., $x_{i}=$ $\mu_{i} \pm h_{i} \sigma_{i}$.

3.4. The Procedure of HDMR-Based Reliability. In this study, HDMR was adopted to approximate the limit state function for reliability analysis of tunnel. Center sample method is used to build the sample points. The response (displacement and plastic zone) was calculated using numerical method and analytical method. The coefficients of component function were determined using moving least square method. FORM
TABLE 1: Statistical property of random variables [17].

\begin{tabular}{lcc}
\hline Rock properties & Mean value & Standard deviation \\
\hline$E(\mathrm{MPa})$ & 373 & 48 \\
$c(\mathrm{MPa})$ & 0.23 & 0.068 \\
$\varphi\left({ }^{\circ}\right)$ & 22.85 & 1.31 \\
\hline
\end{tabular}

was used to estimate the reliability index of tunnel stability. The procedure of HDMR-based reliability analysis is summarized in the flowchart in Figure 1 and explained in detail as follows:

Step 1. Collect the engineering information, such as geological conditions, project scale, rock mechanical parameters, and in situ stress; determine the random variables and component function of HDMR; build a numerical or analytical model.

Step 2 (generate sample points). Central sampling is adopted for generating $2 n+1$ sample points.

Step 3 (build samples set for HDMR). The response of tunnel (displacement, plastic zone, stress and strain, etc.) at each sample point, determined beforehand using analytical or numerical method, is used to determine the limit state function. Sample sets consist of the sample points and their corresponding response.

Step 4 (determine the HDMR-based response surface). The HDMR model was adopted in this study to approximate the limit state function. Moving least square method was used to determine the coefficient of component function in HDMR model.

Step 5 (compute reliability index and design point). The FORM algorithm of Low and Tang (2007) is adopted to determine the reliability index and the design point based on the HDMR-based reliability analysis.

Step 6 (check convergence). Steps 2 to 5 are repeated until the reliability index converges. In this work, convergence is said to occur when the absolute difference between the current and the previous tentative reliability index is less than 0.0001 .

\section{Applications}

In this study, two tunnels in the previous study (Zhao et al., 2014) were revisited to verify and demonstrate the proposed reliability analysis method [17]. For the comparison, the same parameters and model of tunnel were used. The results show the proposed method is efficient and effective to the reliability analysis of rock tunnel. It is feasible to deal with the uncertainty using the proposed method for rock tunnel.

4.1. Circular Tunnel Subjected to Hydrostatic Stress [17]. A circular tunnel is excavated in a continuous, homogeneous, isotropic, initially elastic rock mass subjected to hydrostatic 


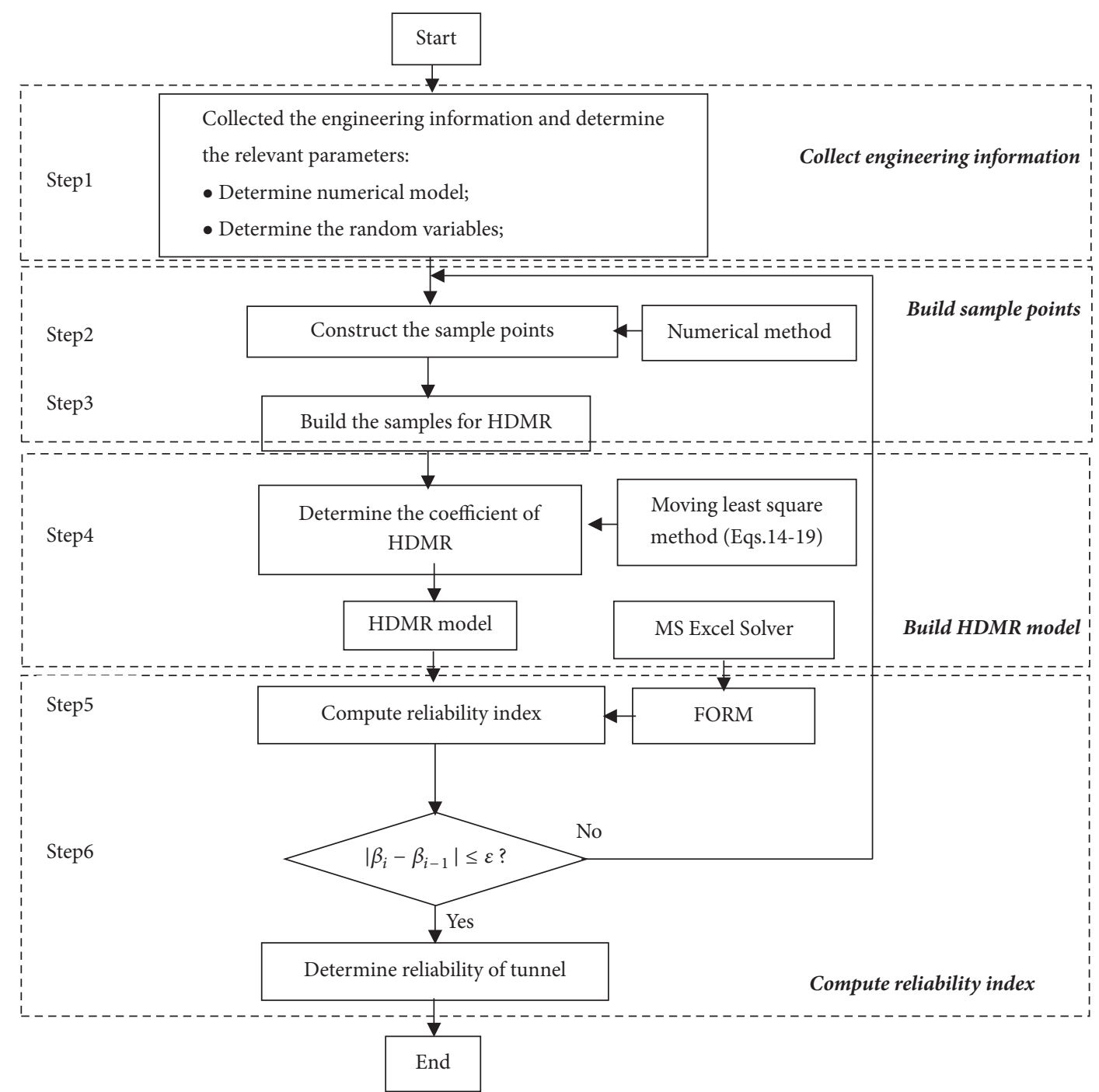

FIgURE 1: The flowchart of the HDMR-based reliability analysis.

far field stress $p_{0}$ and uniform support pressure $p_{i}$ as shown in Figure 2.

If $p_{i}$ is less than the critical pressure $p_{c r}$, a plastic zone exists. In this study, the plastic zone and inward displacement were adopted to estimate the stability of tunnel. According to the Mohr-Coulomb criterion, the plastic zone radius $r_{p}$ and the inward displacement of tunnel wall $u_{i p}$ are given by [26]:

$$
\begin{aligned}
\frac{\mathrm{r}_{\mathrm{p}}}{\mathrm{r}_{0}} & =\left[\frac{2\left(\mathrm{p}_{0}+\mathrm{s}\right)}{(\mathrm{k}+1)\left(\mathrm{p}_{\mathrm{i}}+\mathrm{s}\right)}\right]^{1 /(\mathrm{k}-1)}, \\
\frac{\mathrm{u}_{\mathrm{ip}}}{\mathrm{r}_{0}} & =\left[\frac{1+\mu}{\mathrm{E}}\right]\left[2(1-\mu)\left(\mathrm{p}_{0}-\mathrm{p}_{\mathrm{cr}}\right)\left(\frac{\mathrm{r}_{\mathrm{p}}}{\mathrm{r}_{0}}\right)^{2}\right. \\
& \left.-2(1-2 \mu)\left(\mathrm{p}_{0}-\mathrm{p}_{\mathrm{i}}\right)\right],
\end{aligned}
$$

where $E$ is the elastic modulus and $\mu$ is Poisson's ratio. The values of $p_{c r}, k$, and $s$ are obtained from the following equations:

$$
\begin{aligned}
\mathrm{p}_{\mathrm{cr}} & =\frac{2 \mathrm{p}_{0}-\sigma_{\mathrm{c}}}{\mathrm{k}+1} \\
\mathrm{k} & =\frac{1+\sin \varphi}{1-\sin \varphi} \\
\sigma_{\mathrm{c}} & =\frac{\mathrm{c}(\mathrm{k}-1)}{\tan \varphi}
\end{aligned}
$$

where

$$
\mathrm{s}=\frac{\sigma_{\mathrm{c}}}{\mathrm{k}-1},
$$

$\varphi$ is the friction angle, and $c$ is the cohesion.

In this study, Young's modulus E, cohesion c, and friction angle $\varphi$ of rock mass were the random variables. Normal 
TABLE 2: Reliability comparison of displacement in different support pressures.

\begin{tabular}{|c|c|c|c|c|c|c|c|c|}
\hline \multirow{3}{*}{$p_{i}(\mathrm{Mpa})$} & \multicolumn{4}{|c|}{ HDMR } & \multicolumn{4}{|c|}{ FORM } \\
\hline & \multirow{2}{*}{ Reliability index } & \multicolumn{3}{|c|}{ Design point } & \multirow{2}{*}{ Reliability index } & \multicolumn{3}{|c|}{ Design point } \\
\hline & & $\mathrm{E} / \mathrm{MPa}$ & $\mathrm{c} / \mathrm{MPa}$ & $\varphi /^{\circ}$ & & $\mathrm{E} / \mathrm{MPa}$ & $\mathrm{c} / \mathrm{MPa}$ & $\varphi /^{\circ}$ \\
\hline 0.6 & 0.1457 & 367.3984 & 0.2249 & 22.8493 & 0.1461 & 367.3551 & 0.2252 & 22.8399 \\
\hline 0.8 & 1.9802 & 285.8693 & 0.1879 & 22.6957 & 1.9807 & 286.0296 & 0.1879 & 22.6818 \\
\hline 1.0 & 3.3316 & 215.5761 & 0.2018 & 22.6527 & 3.3315 & 215.7354 & 0.2003 & 22.6645 \\
\hline 1.2 & 4.2080 & 171.0550 & 0.2257 & 22.8258 & 4.2067 & 171.2994 & 0.2210 & 22.7729 \\
\hline
\end{tabular}

TABLE 3: Reliability comparison of plastic zone in different support pressures.

\begin{tabular}{|c|c|c|c|c|c|c|c|c|}
\hline \multirow{3}{*}{$p_{i}(\mathrm{MPa})$} & \multicolumn{4}{|c|}{ HDMR } & \multicolumn{4}{|c|}{ FORM } \\
\hline & \multirow{2}{*}{ Reliability index } & \multicolumn{3}{|c|}{ Design point } & \multirow{2}{*}{ Reliability index } & \multicolumn{3}{|c|}{ Design point } \\
\hline & & $\mathrm{E} / \mathrm{MPa}$ & $\mathrm{c} / \mathrm{MPa}$ & $\varphi /^{\circ}$ & & $\mathrm{E} / \mathrm{MPa}$ & $\mathrm{c} / \mathrm{MPa}$ & $\varphi /^{\circ}$ \\
\hline 0.1 & 1.5313 & 372.9700 & 0.1310 & 23.2682 & 1.5294 & 373.0000 & 0.1328 & 23.1687 \\
\hline 0.15 & 1.9525 & 373.0034 & 0.1042 & 23.3512 & 1.9500 & 373.0000 & 0.1067 & 23.2211 \\
\hline 0.2 & 2.3759 & 373.0990 & 0.0773 & 23.4380 & 2.3720 & 373.0000 & 0.0810 & 23.2569 \\
\hline 0.25 & 2.8002 & 373.0490 & 0.0508 & 23.5009 & 2.7952 & 373.0000 & 0.0555 & 23.2758 \\
\hline
\end{tabular}

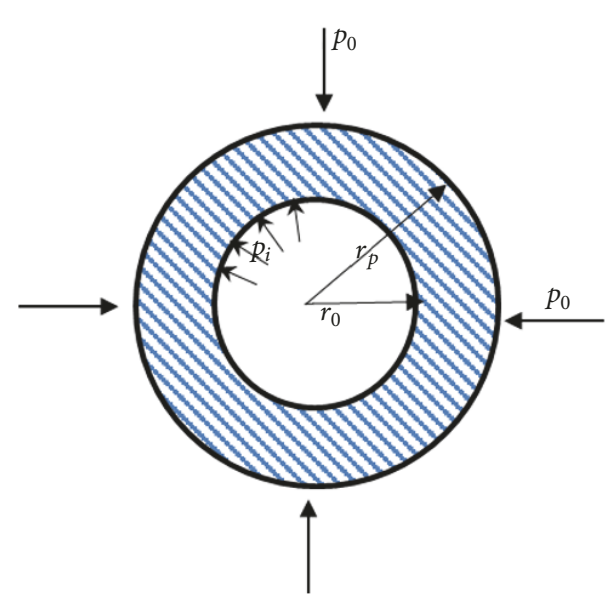

Figure 2: A circular tunnel with hydrostatic stress.

TABLE 4: Parameters of random variables for horseshoe tunnel [17].

\begin{tabular}{lccccc}
\hline & $\mathrm{E}(\mathrm{MPa})$ & $\mathrm{c}(\mathrm{MPa})$ & $\varphi\left(^{\circ}\right)$ & $\sigma_{1}(\mathrm{MPa})$ & $\sigma_{3}(\mathrm{MPa})$ \\
\hline Mean value & 4600 & 1 & 35 & 10 & 7 \\
Standard deviation & 800 & 0.2 & 5 & 2 & 1.4 \\
\hline
\end{tabular}

distribution was assumed for the random variables. The statistical values were listed in Table 1 [17]. In addition, $c$ and $\varphi$ were assumed to be negatively correlated, with a correlation coefficient of -0.5. Radius of tunnel $r_{0}$, hydrostatic stress $p_{0}$, support pressure $p_{i}$, and Poisson's ratio $\mu$ were regarded as deterministic variables. To the stability analysis, $p_{0}=2.5$ $\mathrm{MPa}, p_{i}=0 \mathrm{Mpa}$, and $r_{0}=1 \mathrm{~m}$. Two failure modes were estimated, respectively, i.e., radius of plastic zone and inward displacement. The limit state functions of the two failure modes are given by

$$
\begin{aligned}
& \mathrm{g}_{1}(\mathrm{x})=\mathrm{L}-\frac{\mathrm{r}_{\mathrm{p}}}{\mathrm{r}_{0}}, \\
& \mathrm{~g}_{2}(\mathrm{x})=\varepsilon_{\mathrm{L}}-\frac{\mathrm{u}_{\mathrm{ip}}}{\mathrm{r}_{0}},
\end{aligned}
$$

where $L$ is the permissible threshold about the plastic zone and $\varepsilon_{L}$ is the inwards displacement of tunnel wall. In this study, $L$ and $\varepsilon_{L}$ are 3 and 0.01 , respectively. To illustrate the proposed HDMR-based reliability analysis method, stability analyses were carried out for variations of the circular tunnel case.

The reliability of two failure modes was estimated using the HDMR-based reliability analysis. Figure 3 showed the reliability index and comparison with FORM in different support pressure $\left(p_{0}=2.5 \mathrm{MPa}\right)$. The reliability index of HDMRbased reliability analysis was well agreed with the value of FORM. The comparison between reliability index and design point was listed in Tables 2 and 3. The maximum relative error of the two failure modes was less than $0.3 \%$ and $0.2 \%$, respectively. The maximum relative error of design point was less than $2.2 \%$ to the displacement failure mode. The maximum relative error of design point was less than $8.4 \%$ to the plastic zone failure mode. The results showed the HDMR-based response surface can exactly approximate the response of tunnel. This indicates that the proposed method can replace numerical and analytical method for reliability analysis. Specially, it is important to have reliability analysis for practical rock engineering without analytical solution.

The failure probability of a circular tunnel was analyzed for cases with different support pressures. Figure 4 showed the variation of failure probability with the support pressure. The probability failure decreased with increasing support pressure. It showed the support structure can improve the stability of tunnel and reduce the risk of tunnel failure. 


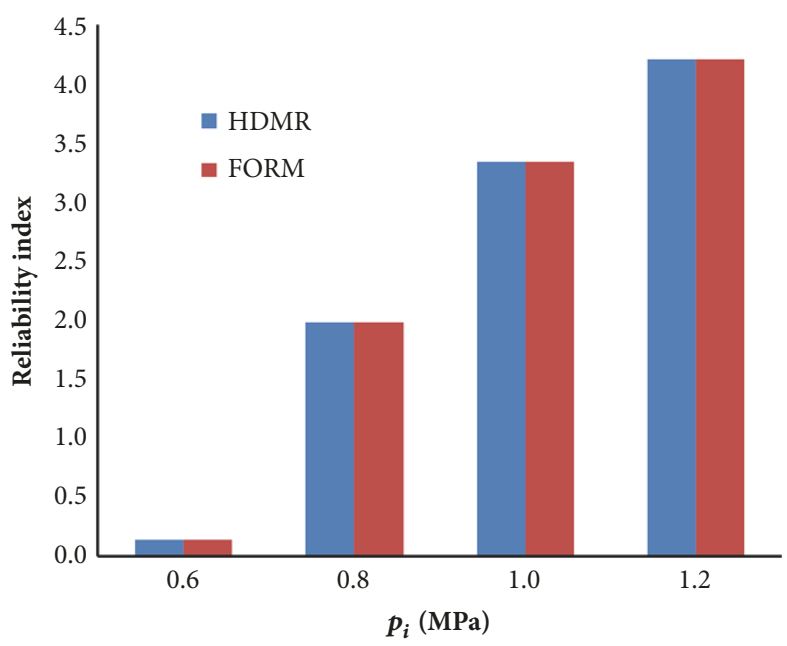

(a) Displacement

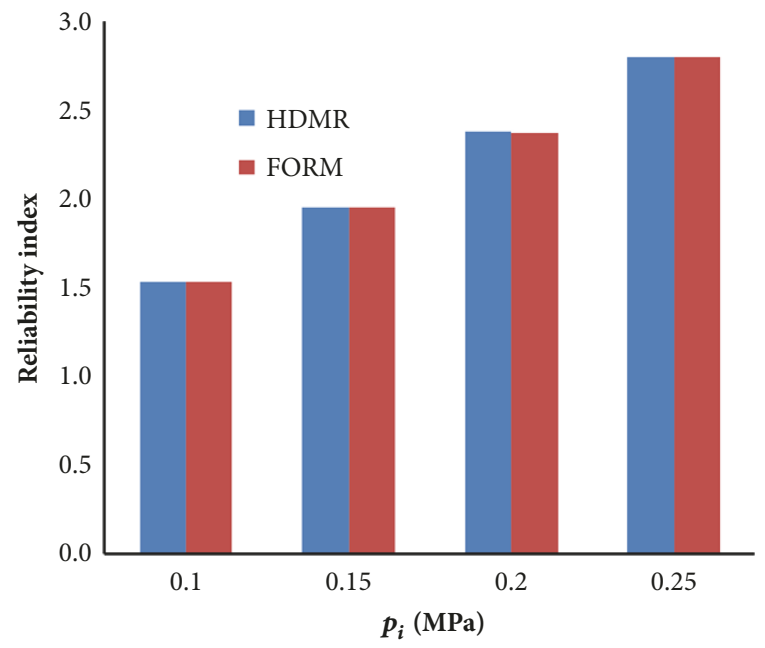

(b) Plastic zone

Figure 3: Comparison of reliability index in different support pressures.

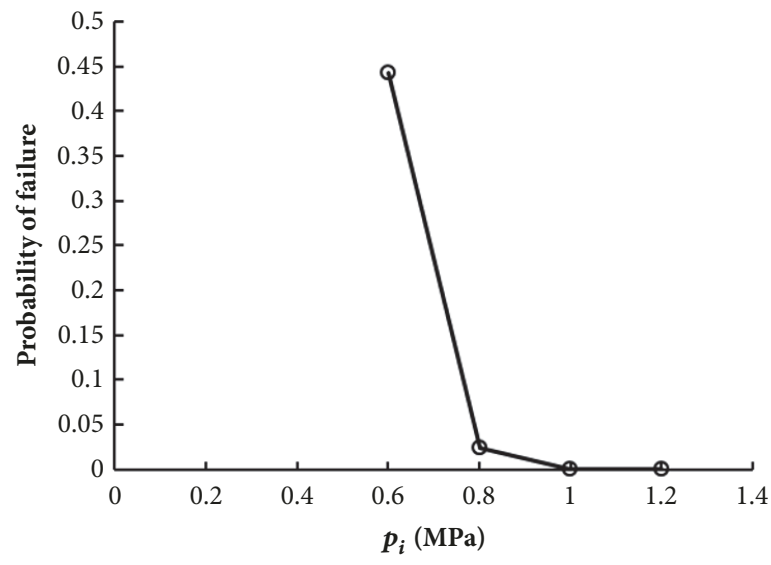

(a) Displacement

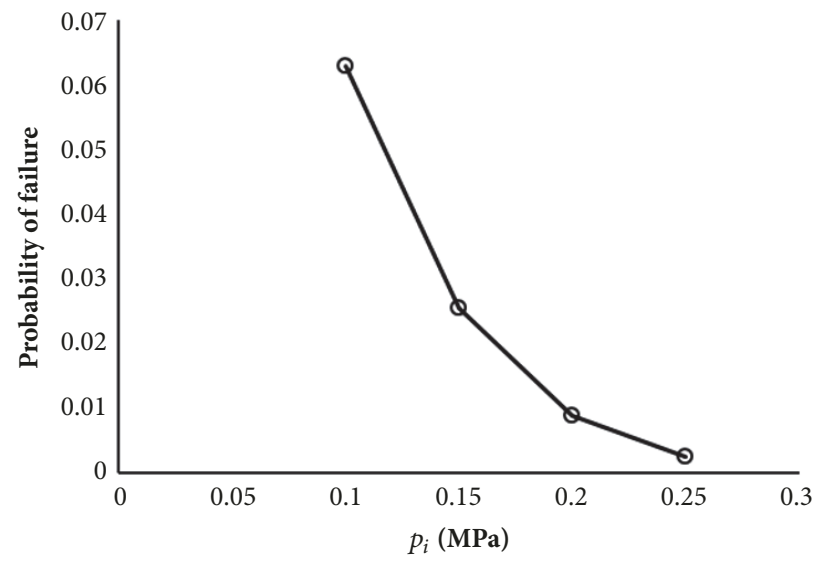

(b) Plastic zone

FIGURE 4: Comparison of failure probability in different support pressures.

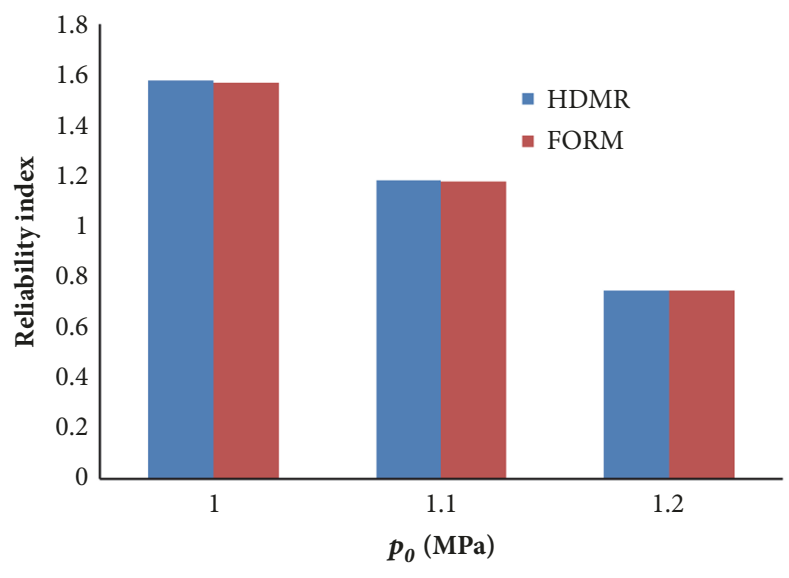

(a) Displacement

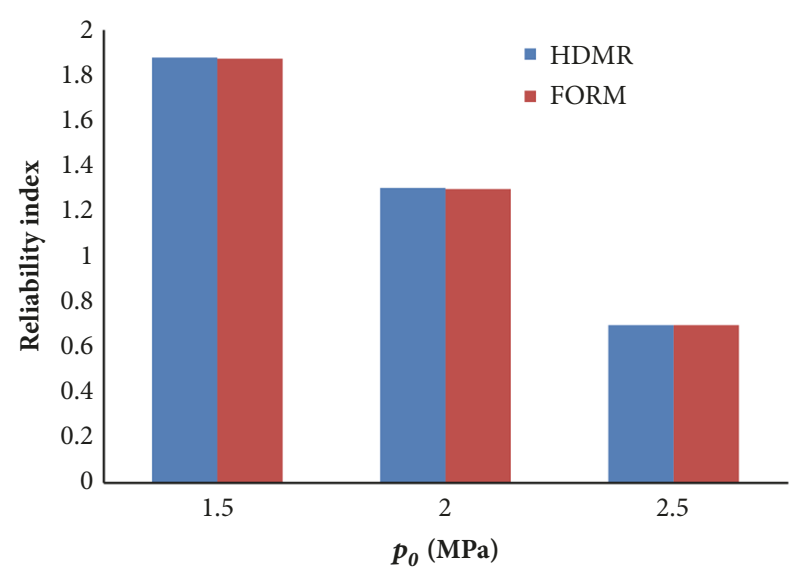

(b) Plastic zone

FIgURE 5: Comparison of reliability index in different far field stress. 


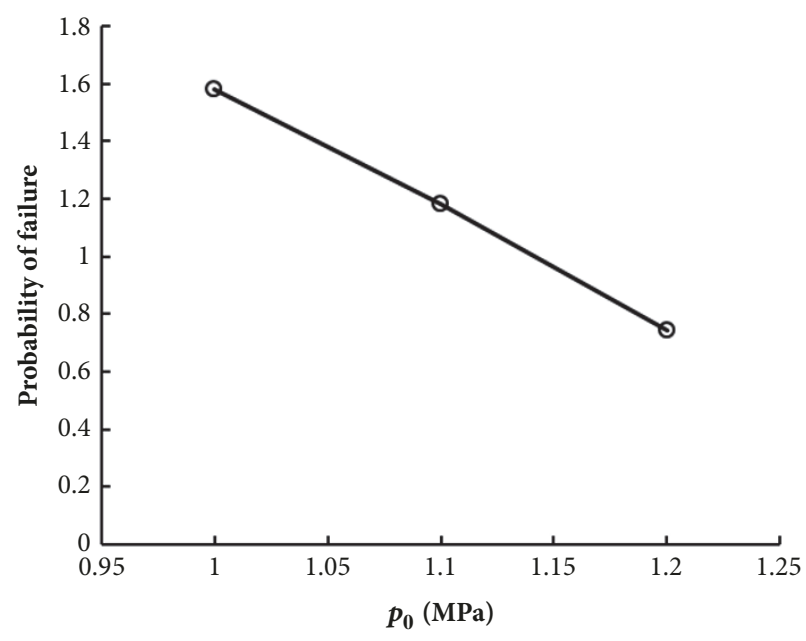

(a) Displacement

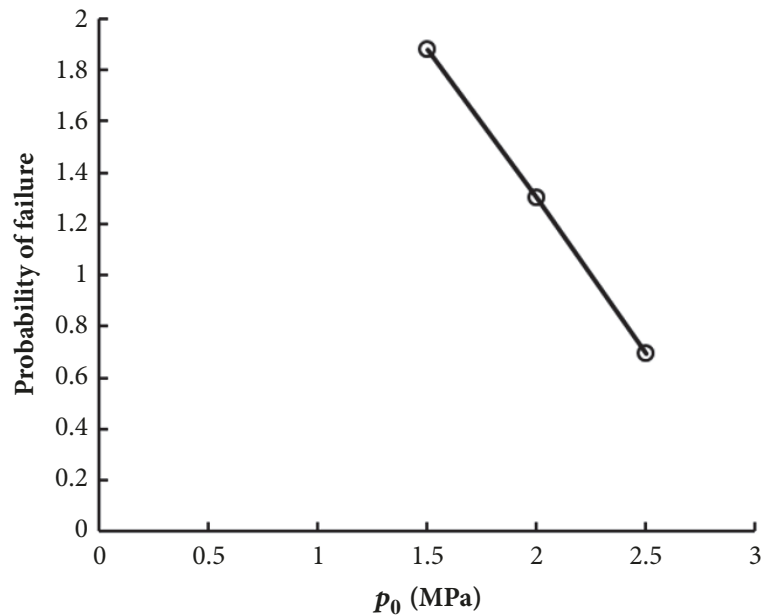

(b) Plastic zone

FIGURE 6: Comparison of failure probability in different far field stresses.

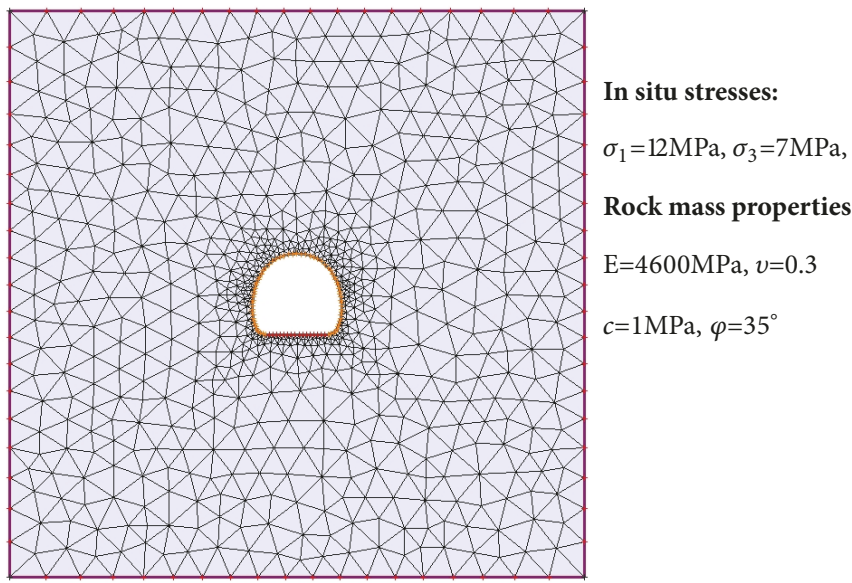

Figure 7: Numerical model of horseshoe tunnel [17].

The stability of tunnel was analyzed for cases with different far field stresses. Figure 5 showed the reliability index and comparison with FORM in different support pressure $\left(p_{i}=0 \mathrm{MPa}\right)$. The reliability index of HDMR-based reliability analysis was very close to the value of FORM. The failure probability of tunnel was shown in Figure 6 in different far field stresses. The probability failure increased with increasing far field stresses. The results of reliability analysis were similar to the results in different support pressure.

4.2. Horseshoe Tunnel [17]. In the above example, HDRMbased reliability analysis was demonstrated based on response surface using a circular tunnel with analytical solution. To a practical tunnel, it is difficult to obtain the analytical solution. Numerical method is commonly used in stability analysis and design of tunnel. In this section, the reliability analysis of a horseshoe-shaped tunnel was estimated using the HDMRbased reliability analysis through combining with numerical method. The span of tunnel is about $5 \mathrm{~m}$ (Figure 7). The elastic modulus $E(\mathrm{MPa})$, friction angle $\varphi\left({ }^{\circ}\right)$, cohesion $c(\mathrm{MPa})$, major principal stress $\sigma_{1}(\mathrm{MPa})$, and minor principal stress $\sigma_{3}(\mathrm{MPa})$ were regarded as random variables [17]. The in situ stress and Poisson's ratio were the deterministic. The mean value of the rock mass parameters and in situ stress are shown in Figure 7. The statistical properties of random variables are listed in Table 4. The inward displacement of the tunnel wall was selected as the criterion of the stability of the tunnel. A two-dimensional finite element model of the tunnel was used to compute the response (displacement) of tunnel (Figure 7).

The reliability index and design point were listed in Table 5 . The reliability index obtained by the HDMR-based reliability analysis is 2.336 and is agreed very closely with the values (2.341) obtained by RSM (Zhao et al., 2014), with a relative error of about $0.21 \%$. The design points were also close to the results of RSM, and the maximum relative error is less than 6\%. These showed the HDMR-based response surface can approximate well the response of tunnel instead of numerical method. It provided a good way to apply reliability analysis for practical tunnel engineering. 
TABLE 5: The results and comparison of reliability analysis for horseshoe tunnel.

\begin{tabular}{lccccccc}
\hline \multirow{2}{*}{ Method } & \multirow{2}{*}{ Reliability index } & $\mathrm{E}(\mathrm{MPa})$ & $\mathrm{c}(\mathrm{MPa})$ & $\varphi\left(^{\circ}\right)$ & $\sigma_{1}(\mathrm{MPa})$ & $\sigma_{3}(\mathrm{MPa})$ & Number of iteration \\
& & 3200.172 & 1.002 & 31.446 & 12.633 & 6.994 & 4 \\
\hline HDMR-RSM & 2.336 & 3338.681 & 0.945 & 33.072 & 13.195 & 7.109 & 5 \\
Polynimial RSM & 2.341 & 3125.139 & 0.990 & 32.178 & 12.564 & 6.915 & 4 \\
LSSVM-RSM & 2.347 & 4.149 & 5.983 & 4.916 & 4.263 & 2.325 & - \\
Relative error (\%) & 0.214 & & & & \\
\hline
\end{tabular}

\section{Conclusions}

In this study, HDMR was adopted to approximate the response of tunnel, and that combined with response surface method to build the limit state function. HDMR use the low dimensional function to present the complex, implicit, high dimensional, and nonlinear relationship between random variables and response of tunnel. FORM was used to compute the reliability index. The HDMR-based reliability analysis was applied to two tunnels. The reliability index and design points were well agreed with the FORM and RSM. HDMR can replace numerical method in reliability analysis. This is important to practical tunnel that it is difficult to obtain the analytical solution of tunnel response. The results showed the proposed method is useful to reliability analysis for practical tunnel engineering. HDMR-based reliability analysis provided a good tool to consider the uncertainty for practical rock engineering.

\section{Data Availability}

The data used to support the findings of this study are available from the corresponding author upon request.

\section{Conflicts of Interest}

The authors declare no conflicts of interest.

\section{Acknowledgments}

The authors would like to gratefully acknowledge that this research study was supported by the Program for Innovative Research Team (Science and Technology) of the University of Henan Province (No. 15IRTSTHN029).

\section{References}

[1] E. Hoek, "Reliability of Hoek-Brown estimates of rock mass properties and their impact on design," International Journal of Rock Mechanics and Mining Sciences, vol. 35, no. 1, pp. 63-69, 1998.

[2] J. Deng, D. Gu, X. Li, and Z. Q. Yue, "Structural reliability analysis for implicit performance functions using artificial neural network," Structural Safety, vol. 27, no. 1, pp. 25-48, 2005.

[3] P. Oreste, "A probabilistic design approach for tunnel supports," Computers \& Geosciences, vol. 32, no. 7, pp. 520-534, 2005.
[4] G. Mollon, D. Dias, and A.-H. Soubra, "Probabilistic analysis of pressurized tunnels against face stability using collocationbased stochastic response surface method," Journal of Geotechnical and Geoenvironmental Engineering, vol. 137, no. 4, pp. 385397, 2011.

[5] G. Mollon, D. Dias, and A.-H. Soubra, "Probabilistic analysis of circular tunnels in homogeneous soil using response surface methodology," Journal of Geotechnical and Geoenvironmental Engineering, vol. 135, no. 9, pp. 1314-1325, 2009.

[6] H.-Z. Li and B. K. Low, "Reliability analysis of circular tunnel under hydrostatic stress field," Computers \& Geosciences, vol. 37, no. 1-2, pp. 50-58, 2010.

[7] Q. Lü and B. K. Low, "Probabilistic analysis of underground rock excavations using response surface method and SORM," Computers \& Geosciences, vol. 38, no. 8, pp. 1008-1021, 2011.

[8] Q. Lü, Z.-P. Xiao, J. Ji, and J. Zheng, "Reliability based design optimization for a rock tunnel support system with multiple failure modes using response surface method," Tunnelling and Underground Space Technology, vol. 70, pp. 1-10, 2017.

[9] Q. Lü, H.-Y. Sun, and B. K. Low, "Reliability analysis of groundsupport interaction in circular tunnels using the response surface method," International Journal of Rock Mechanics and Mining Sciences, vol. 48, no. 8, pp. 1329-1343, 2011.

[10] Y.-H. Su, X. Li, and Z.-Y. Xie, "Probabilistic evaluation for the implicit limit-state function of stability of a highway tunnel in China," Tunnelling and Underground Space Technology, vol. 26, no. 2, pp. 422-434, 2011.

[11] A. H. Elhewy, E. Mesbahi, and Y. Pu, "Reliability analysis of structures using neural network method," Probabilistic Engineering Mechanics, vol. 21, no. 1, pp. 44-53, 2006.

[12] J. Cheng and Q. S. Li, "Reliability analysis of structures using artificial neural network based genetic algorithms," Computer Methods Applied Mechanics and Engineering, vol. 197, no. 45-48, pp. 3742-3750, 2008.

[13] J. B. Cardoso, J. R. de Almeida, J. M. Dias, and P. G. Coelho, "Structural reliability analysis using Monte Carlo simulation and neural networks," Advances in Engineering Software, vol. 39, no. 6, pp. 505-513, 2008.

[14] P. A. M. Lopes, H. M. Gomes, and A. M. Awruch, "Reliability analysis of laminated composite structures using finite elements and neural networks," Composite Structures, vol. 92, no. 7, pp. 1603-1613, 2010.

[15] Q. Lü, C. L. Chan, and B. K. Low, "Probabilistic evaluation of ground-support interaction for deep rock excavation using artificial neural network and uniform design," Tunnelling and Underground Space Technology, vol. 32, pp. 1-18, 2012.

[16] H. B. Zhao, "Slope reliability analysis using a support vector machine," Computers \& Geosciences, vol. 35, no. 3, pp. 459-467, 2008.

[17] H. Zhao, Z. Ru, X. Chang, S. Yin, and S. Li, "Reliability analysis of tunnel using least square support vector machine," Tunnelling 
and Underground Space Technology, vol. 41, no. 1, pp. 14-23, 2014.

[18] H. Rabitz and Ö. F. Aliş, "General foundations of highdimensional model representations," Journal of Mathematical Chemistry, vol. 25, no. 2-3, pp. 197-233, 1999.

[19] A. M. Hasofer and N. C. Lind, "Exact and invariant secondmoment code format," Journal of the Engineering Mechanical Division, vol. 100, no. 1, pp. 111-121, 1974.

[20] B. K. Low and W. H. Tang, "Reliability analysis using objectoriented constrained optimization," Structural Safety, vol. 26, no. 1, pp. 69-89, 2004.

[21] B. K. Low and W. H. Tang, "Efficient spreadsheet algorithm for first-order reliability method," Journal of Engineering Mechanics, vol. 133, no. 12, pp. 1378-1387, 2007.

[22] O. F. Alis and H. Rabitz, "Efficient implementation of high dimensional model representations," Journal of Mathematical Chemistry, vol. 29, no. 2, pp. 127-142, 2001.

[23] G. Li, S.-W. Wang, C. Rosenthal, and H. Rabitz, "High dimensional model representations generated from low dimensional data samples. I. mp-Cut-HDMR," Journal of Mathematical Chemistry, vol. 30, no. 1, pp. 1-30, 2001.

[24] P. Lancaster and K. Salkauskas, Curve and surface fitting: An Introduction, Academic Press, London, UK, 1986.

[25] Q. Lü, Z.-P. Xiao, J. Ji, J. Zheng, and Y.-Q. Shang, "Moving least squares method for reliability assessment of rock tunnel excavation considering ground-support interaction," Computers \& Geosciences, vol. 84, pp. 88-100, 2017.

[26] M. E. D. Fama, "Numerical modelling of yield zones in weak rocks," in Comprehensive Rock Engineering, J. A. Hudson, Ed., vol. 2, pp. 49-75, McGraw Hill, Oxford, UK, 1993. 


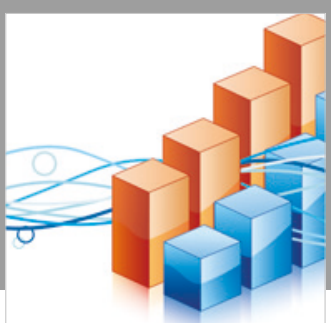

Advances in

Operations Research

\section{-n-m}
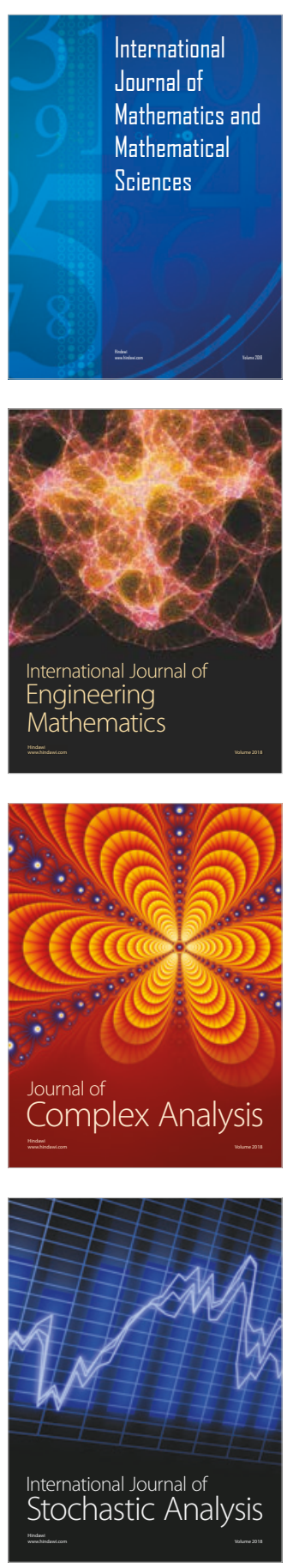
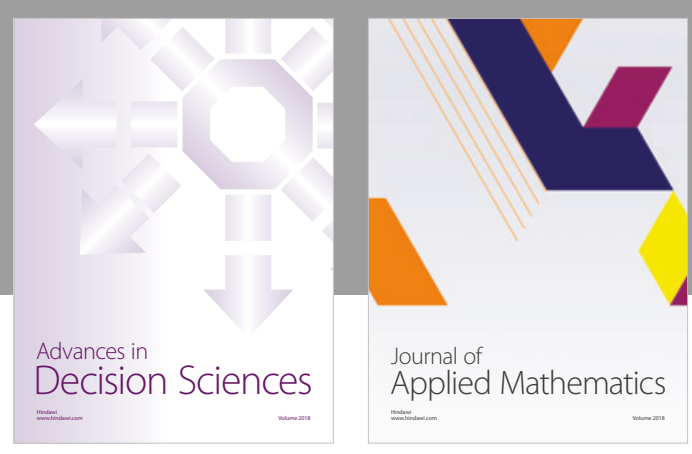

Journal of

Applied Mathematics
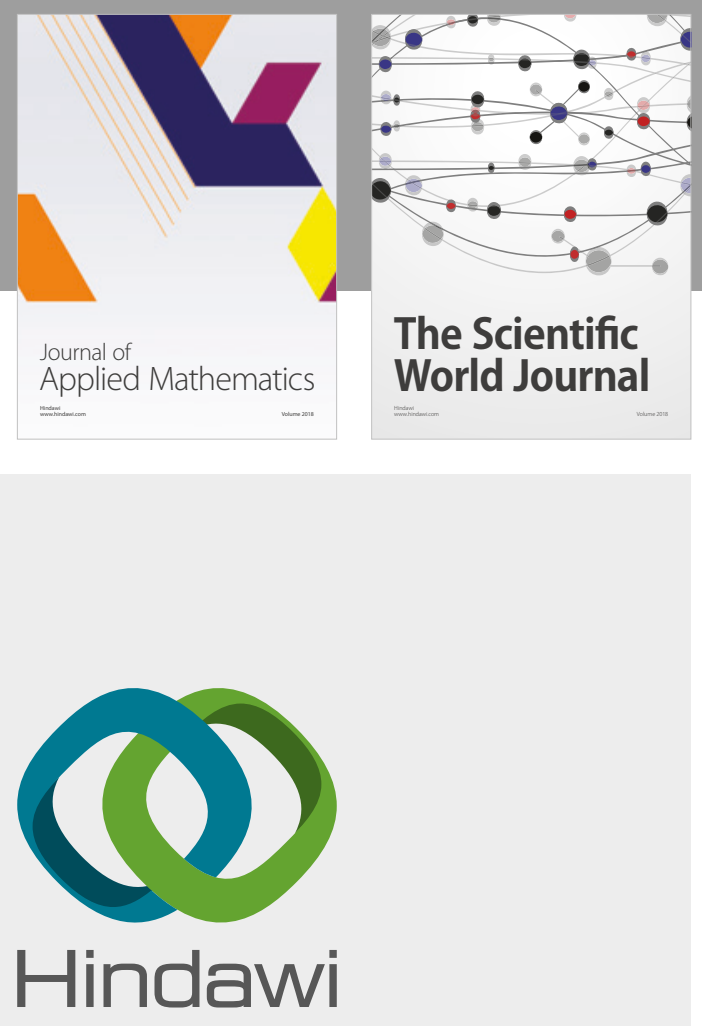

Submit your manuscripts at

www.hindawi.com

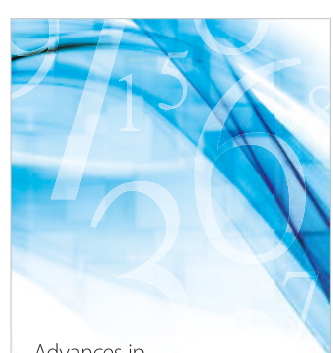

Advances in
Numerical Analysis
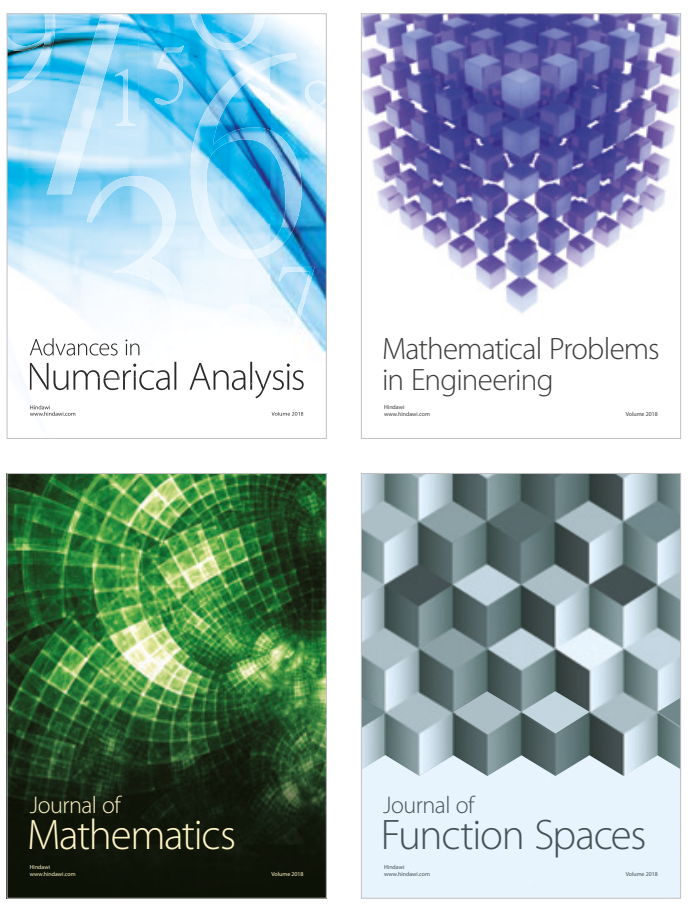

Mathematical Problems in Engineering

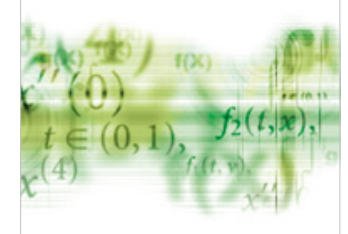

International Journal of

Differential Equations

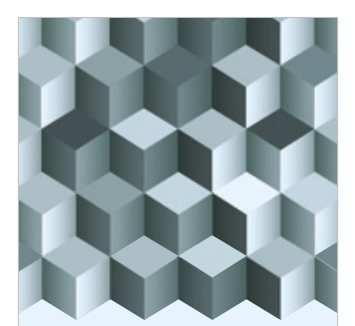

Journal of

Function Spaces

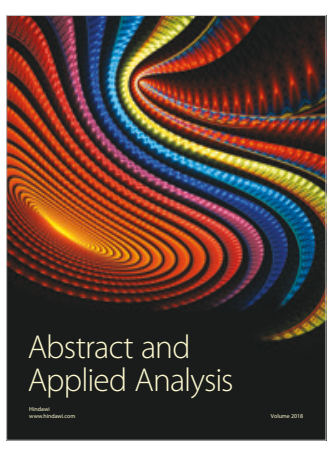

The Scientific

World Journal

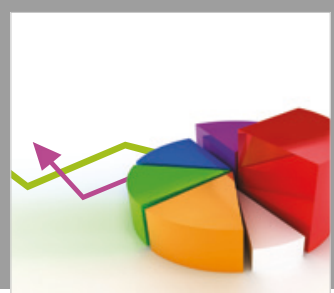

Journal of

Probability and Statistics
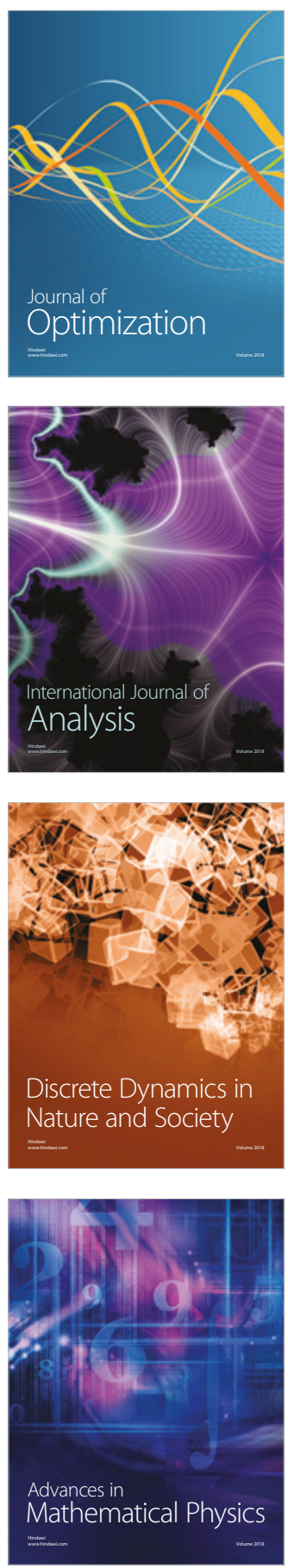